\title{
A novel ARMS-based assay for the quantification of EGFR mutations in patients with lung adenocarcinoma
}

\author{
YAZHEN ZHU ${ }^{1 *}$, ZHIWEI GUO $^{2 *}$, YING LIU ${ }^{1}$, XIYUN ZHENG $^{1}$, GUOHUA YANG $^{2}$ and GUANGJUAN ZHENG ${ }^{1}$ \\ ${ }^{1}$ Department of Pathology, Guangdong Provincial Hospital of TCM, Guangzhou University of Chinese Medicine, \\ Guangdong Provincial Academy of Chinese Medical Sciences, Guangzhou, Guangdong 510120; \\ ${ }^{2}$ GenoSaber Biotech Co., Ltd., Shanghai 201203, P.R. China
}

Received July 26, 2017; Accepted November 21, 2017

DOI: $10.3892 / \mathrm{ol} .2017 .7679$

\begin{abstract}
Quantification of epidermal growth factor receptor $(E G F R)$ mutations is important for the prediction of tyrosine kinase inhibitor (TKI) efficacy in patients with non-small cell lung cancer (NSCLC). However, clinicians lack a sensitive and convenient method to quantify EGFR mutant abundance. The present study introduces a novel method, namely amplification refractory mutation system (ARMS)-Plus, for the quantitative analysis of EGFR exon 19 deletion (19Del), L858R and $T 790 M$ mutations. Formalin-fixed paraffin-embedded tumor samples were collected from 77 patients with lung adenocarcinoma. DNA was extracted and analyzed for EGFR mutations using ARMS-Plus. The performance of ARMS-Plus was then compared with that of conventional ARMS-polymerase chain reaction (ARMS-PCR) and droplet digital PCR (ddPCR). The results demonstrated that the concordance rate of EGFR

Correspondence to: Dr Guohua Yang, GenoSaber Biotech Co., Ltd., Block 26, Lane 908 Ziping Road, Pudong, Shanghai 201203, P.R. China

E-mail: ghyang@genosaber.cn

Dr Guangjuan Zheng, Department of Pathology, Guangdong Provincial Hospital of TCM, Guangzhou University of Chinese Medicine, Guangdong Provincial Academy of Chinese Medical Sciences, 111 Dade Road, Guangzhou, Guangdong 510120, P.R. China E-mail: zhengguangjuan@163.com
\end{abstract}

Abbreviations: ARMS, amplification refractory mutation system; CFDA, China Food and Drug Administration; PCR, polymerase chain reaction; ddPCR, droplet digital PCR; EGFR, epidermal growth factor receptor; 19Del, exon 19 deletion; FFPE, formalin-fixed paraffin-embedded; MAPK, mitogen-activated protein kinase; NSCLC, non-small cell lung cancer; PFS, progression-free survival; SNP, single nucleotide polymorphism; TKIs, tyrosine kinase inhibitors

${ }^{*}$ Contributed equally

Key words: epidermal growth factor receptor, non-small cell lung cancer, amplification refractory mutation system, droplet digital polymerase chain reaction, formalin-fixed paraffin-embedded mutation testing between ARMS-Plus and ddPCR was $98.7 \%$ (76/77, Kappa=0.9739). 19Del and L858R mutations were detected in 23 and 12 patients, respectively. There was a significant difference between ARMS-Plus and ddPCR in the evaluation of 19Del mutant abundance $(\mathrm{P}=0.0002)$; however, not in that of $L 858 R$ mutant abundance $(\mathrm{P}=0.7334)$. The ARMS-Plus results in $L 858 R$ mutant abundance were concordant with that of ddPCR $\left(R^{2}=0.8081\right)$. These results indicated that the sensitivity and specificity of ARMS-Plus in identifying EGFR mutations were similar to that of ddPCR. For quantitative analysis, the results of ARMS-Plus in evaluating L858R mutant abundance revealed a positive correlation with the ddPCR results. Thus, ARMS-Plus provides an alternative method, which is reliable and cost-effective, to quantify EGFR mutations and thereby, aid treatment decisions in patients with lung adenocarcinoma.

\section{Introduction}

Lung cancer, being the leading cause of cancer-related mortality, results in over 1 million deaths per year worldwide. Non-small cell lung cancer (NSCLC) accounts for about $85 \%$ of lung cancer cases. The prognosis of NSCLC is poor, with a 5 -year overall survival rate of less than $20 \%$ (1). Epidermal growth factor receptor $(E G F R)$ is a transmembrane receptor tyrosine kinase frequently over-expressed in NSCLC (2). Activation of EGFR induces the proliferation and growth of cancer cells and thus EGFR is a promising target for personalized therapy. Recently, EGFR-tyrosine kinase inhibitor (TKI) such as Gefitinib (AstraZeneca, London, UK), Erlotinib (Genentech, San Francisco, USA) and Afatinib (Boehringer Ingelheim, Ingelheim, Germany) have emerged as the first-line therapy for the treatment of NSCLC (3-5). Through selectively binding at the ATP-binding site of the receptor kinase domain, TKIs block EGFR activation and its downstream signal transduction, such as STAT3, MAP kinase (MAPK) and AKT signaling (6). Although the EGFR-TKIs show a rapid and dramatic tumor regression in NSCLC patients, the initial clinical study with Gefitinib in 2005 illustrated that only 9-19\% of unselected patients with advanced NSCLC showed responses to the treatment $(7,8)$. Subsequently, researchers reported that EGFR mutation status may be a key predictive factor in determining the 
efficacy of EGFR-TKIs treatment (9). Exon 19 deletions (19Del) and $L 858 R$ substitution mutations were found to be the most common EGFR mutations, which constitute about 90\% of all EGFR mutations (10). Several studies revealed that NSCLC patients with EGFR mutations showed better prognosis on EGFR-TKIs therapy. This was validated by two phase II clinical trials, which demonstrated an overall response rate of $75-78 \%$ for Gefitinib in NSCLC patients harboring EGFR mutations $(11,12)$. Nevertheless, secondary mutations frequently arise after first-generation EGFR-TKIs treatment and lead to therapeutic resistance. T790M mutation is the major cause of resistance to first-generation EGFR-TKIs, accounting for about $70 \%$ of acquired resistance (13). T790M mutation was reported to associate with a shorter progression-free survival (PFS) with EGFR-TKIs therapy in NSCLC patients (14). The relative EGFR mutant abundance may also be a key factor in predicting the sensitivity of EGFR-TKIs. Zhou et al reported that advanced NSCLC patients with a high abundance of EGFR mutations showed a significant longer PFS after Gefitinib treatment (15). In another study, Zhao et al demonstrated that the abundance of $E G F R$ mutations could be a possible predictor to select patients with acquired resistance to primary TKIs that would benefit from EGFR-TKIs re-administration (16). Since the afore-mentioned $E G F R$ mutations are critical for deciding the treatment strategies in patients with NSCLC, a reliable method to identify and measure the EGFR mutant abundance is urgently needed.

In the past decade, direct DNA sequencing was the most frequently used method for EGFR mutation detection. Despite its accessibility, the sensitivity is relatively low. The detection limit of direct DNA sequencing is approximately $20 \%$, which may lead to a high rate of false negative result (17). More importantly, performing direct DNA sequencing requires a long processing time and could delay clinical-decision making. Currently, numerous detection methods have been investigating for the detection of $E G F R$ mutations with a higher sensitivity $(18,19)$. Amplification refractory mutation system (ARMS) is a detection method based on allele-specific polymerase chain reaction (PCR). The detection limit is reported to be around $1 \%$ which is far more sensitive than direct DNA sequencing. Droplet digital PCR (ddPCR) is another emerging method to detect EGFR mutations. Based on the compartmentalization and amplification of single DNA molecules, ddPCR is highly sensitive. Thus, it is now generally accepted as the most sensitive method for the detection and quantification of mutations (20). However, this method requires specialized equipment and it is more expensive in terms of cost and labor. Here, we introduce a modified ARMS-based assay, namely ARMS-Plus, for the analysis of EGFR mutations based on real time PCR platform. In the present study, ARMS-Plus and ddPCR methods were used to analyze FFPE-derived DNA samples, with the aim of comparing the performances of the two methods and exploring the feasibility of using ARMS-Plus in the measuring of EGFR mutant abundance.

\section{Materials and methods}

Sample collection and processing. FFPE tissue samples obtained from patients between 2013 and 2015 were collected from the Guangdong Provincial Hospital of Traditional Chinese Medicine. Written informed consent was obtained from all of the patients. The present study protocol was approved by the Institutional Review Board of the Guangdong Provincial Hospital of Traditional Chinese Medicine. The FFPE tissue samples were micro-dissected by a pathologist and assessed by hematoxylin and eosin staining. Genomic DNA was extracted using the QIAamp ${ }^{\circledR}$ DNA FFPE Tissue kit (Qiagen, Shanghai, China). Collected FFPE-derived DNA samples were then analyzed by the following assays.

ARMS-plus. The isolated DNA samples were processed by Human EGFR Gene Mutation Quantitative Detection kit (Fluorescenece qPCR; GenoSaber Biotech Co., Ltd., Shanghai, China) according to manufacturer's instructions. This is a quantitative PCR kit that can detect 4 different $E G F R$ mutations with an external control in exon 4 to monitor samples qualities (Table I). In brief, $5 \mu 1$ samples were added to the pre-mixed $45 \mu 1$ reaction mixture came with the kit and the PCR was conducted with Applied Biosystems ${ }^{\circledR} 7500$ Real-Time PCR Systems (Thermo Fisher Scientific, Inc., Waltham, MA, USA). PCR amplification conditions were as follow: hot start at $95^{\circ} \mathrm{C}$ for $4 \mathrm{~min}$, and 50 cycles of $95^{\circ} \mathrm{C}$ for $10 \mathrm{sec}, 61^{\circ} \mathrm{C}$ for $30 \mathrm{sec}$ with fluorescence reading (FAM). Primer and probe sequences were listed in Table II. 19Del mutation was detected by two separate reactions as two different kinds of base pair alteration were found. We also found that the 2361-site single nucleotide polymorphism (SNP) may affect PCR specificity, hence the T790M test was divided into two reactions to raise detection rate. The mutant abundance was defined as the percentage of mutant copies present in the whole DNA sample. To calculate the copy number, serially diluted plasmids were provided in the kit as standard samples for generating the standardization curve. Theoretically, the calculation equation of mutant abundance is shown as follow:

$$
\text { Mutant abundance }=\frac{\text { Observed mutant copy number }}{\text { Observed wild type copy number }}
$$

$d d P C R$. ddPCR was performed as previously described (21). In brief, the mixture of probes, primers, template DNA and $2 \mathrm{X}$ Droplet PCR Supermix (Bio-Rad Laboratories, Hercules, CA, USA) was applied to the droplet generator. The oil-in-water mixture generated was then transferred to a 96-well PCR plate, heat-sealed and placed in a C1000 Touch thermal cycler (Bio-Rad Laboratories) for PCR amplification. The following thermo-cycling conditions were used: Hot start at $95^{\circ} \mathrm{C}$ for $10 \mathrm{~min}, 40$ cycles of $94^{\circ} \mathrm{C}$ for $30 \mathrm{sec}, 62^{\circ} \mathrm{C}$ for $1 \mathrm{~min}$, and $98^{\circ} \mathrm{C}$ for $10 \mathrm{~min}$. The PCR product was read on a QX-200 droplet reader (Bio-Rad Laboratories) and the data were analyzed with Quantilife software. The Qunatilife software calculates the copy number of both mutant and wild-type DNA based on the Poisson statistic.

ARMS-PCR. The isolated DNA samples were re-tested with ADx EGFR Mutations Detection kit (Amoy Diagnostics, Xiamen, China), which has received China Food and Drug Administration's (CFDA) approval for clinical usage in mainland China. The kit covered the 29 EGFR mutation hotspots from exon 18 to 21 . The assay was carried out according to 
Table I. Mutations or gene fragments detected by ARMS-Plus.

\begin{tabular}{lcccc}
\hline Site & Mutation & Exon & Base pair altered & Cosmic ID \\
\hline EGFR external control & $/$ & 4 & $/$ & $/$ \\
Del(1) & E746_A750del(1) & 19 & $2235 \_2249$ del15 & 6223 \\
Del(2) & E746_A750del(2) & 19 & $2236 \_2250 d e 115$ & 6225 \\
L858R & L858R & 21 & $2573 T>\mathrm{G}$ & 6224 \\
T790M(1) & $T 790 M$ & 20 & $2369 \mathrm{C}>\mathrm{T}$ & 6240 \\
T790M(2) & $T 790 M$ & 20 & $2361 \mathrm{G}>\mathrm{A} / 2369 \mathrm{C}>\mathrm{T}$ & $1451600 / 6240$
\end{tabular}

The mutant abundance of 19Del mutation is the sum of $\operatorname{Del}(1)$ and $\operatorname{Del}(2)$, while the one with a larger value between T790M(1) and T790M(2) was chosen as the T790M mutant abundance. COSMIC, catalogue of somatic mutations in cancer; EGFR, epidermal growth factor receptor; 19Del, exon 19 deletion; ARMS, amplification refractory mutation system.

Table II. Sequence of primers and probes utilized for ARMS-Plus.

\begin{tabular}{|c|c|c|c|}
\hline Site & Primer & Probe & Blocker \\
\hline $\begin{array}{l}\text { EGFR external } \\
\text { control }\end{array}$ & $\begin{array}{l}\text { Forward: 5'-TGGAGAGCAT } \\
\text { CCAGT-3' } \\
\text { Reverse: 5'-TCTGGAAGTC } \\
\text { CATCGACAT-3' }\end{array}$ & $\begin{array}{l}\text { 5'FAM-ACATAGTCAG } \\
\text { CAGTGACTT-3'MGB }\end{array}$ & I \\
\hline $\operatorname{Del}(1)$ & $\begin{array}{l}\text { Forward: 5'-CCGTCGCTAT } \\
\text { CAAA-3' } \\
\text { Reverse: 5'-ATGGACCCCC } \\
\text { ACAC-3' }\end{array}$ & $\begin{array}{l}\text { 5'FAM-AAGCCAACAA } \\
\text { GGAAAT-3'MGB }\end{array}$ & I \\
\hline $\operatorname{Del}(2)$ & $\begin{array}{l}\text { Forward: 5'-GTCGCTATCA } \\
\text { AGA-3' } \\
\text { Reverse: 5'-GTCGCTATCA } \\
\text { AGA-3' }\end{array}$ & $\begin{array}{l}\text { 5'FAM-AAGCCAACAA } \\
\text { GGAAAT-3'MGB }\end{array}$ & I \\
\hline L858R & $\begin{array}{l}\text { Forward: 5'-AGATTTTGGG } \\
\text { CG-3' } \\
\text { Reverse: 5'-TTTGCCTCCT } \\
\text { TCTGC-3' }\end{array}$ & $\begin{array}{l}\text { 5'FAM-AACTGCTGGG } \\
\text { TGCGGA-3'MGB }\end{array}$ & $\begin{array}{l}\text { 5'-TTTTTGGGCGGGCCA } \\
\text { AAC-3' phosphorthioated }\end{array}$ \\
\hline T790M(1) & $\begin{array}{l}\text { Forward: 5'-CCGTGCAGCA } \\
\text { CATCAT-3' } \\
\text { Reverse: 5'-TGTCTTTGTG } \\
\text { TTCCCG-3' }\end{array}$ & $\begin{array}{l}\text { 5'FAM-CTCATGCCCT } \\
\text { TCGGC-3'MGB }\end{array}$ & $\begin{array}{l}\text { 5'-GCTCATCACGCAGCTCA-3' } \\
\text { phosphorthioated }\end{array}$ \\
\hline $\mathrm{T} 790 \mathrm{M}(2)$ & $\begin{array}{l}\text { Forward: 5'-CCGTGCAACA } \\
\text { CATCAT-3' } \\
\text { Reverse: 5'-TGTCTTTGTG } \\
\text { TTCCCG-3' }\end{array}$ & $\begin{array}{l}\text { 5'FAM-CTCATGCCCT } \\
\text { TCGGC-3'MGB }\end{array}$ & $\begin{array}{l}\text { 5'-CAACTCATCACGCAGCT-3' } \\
\text { phosphorthioated }\end{array}$ \\
\hline
\end{tabular}

EGFR, epidermal growth factor receptor; ARMS, amplification refractory mutation system.

the manufacturer's protocol with the MX3000P (Stratagene; Agilent Technologies, Inc., Santa Clara, CA, USA) Real-Time PCR system.

Statistical analysis. The concordance rate of ARMS-Plus and ddPCR in identifying EGFR mutation status was analyzed by Kappa test. Samples were examined to determine whether a statistical difference existed regarding variations in $E G F R$ mutations between ARMS-Plus and ddPCR by the Wilcoxon test. $\mathrm{P}<0.05$ was considered to indicate statistical significance. The correlation between ARMS-Plus and ddPCR results on the EGFR mutant abundance was examined by Linear Regression analysis. The statistical analysis and graphic generation were carried out by using SAS9.2 (SAS Institute, Inc., Cary, NC, USA) and GraphPad Prism 5 (GraphPad Software, Inc., La Jolla, CA, USA). 

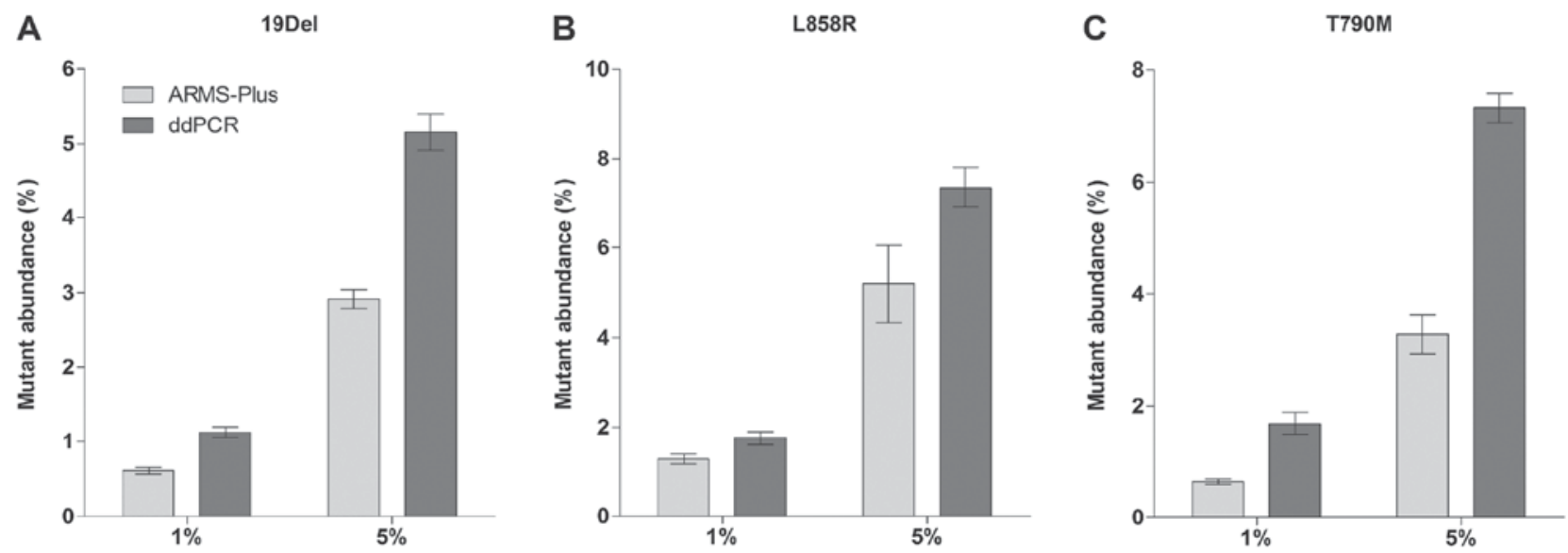

Figure 1. Detection of EGFR mutations by ARMS-Plus and ddPCR in spiked cell mixtures. Mutant cell lines were spiked into a $E G F R$ wild-type cell line at a ratio of 1 and 5\%. The mutant abundance was then detected. EGFR (A) 19Del, (B) L858R, and (C) T790M mutations were stably detected by ARMS-Plus and ddPCR assays. Data are presented as the mean \pm standard error of the mean. EGFR, epidermal growth factor receptor; ARMS, amplification refractory mutation system; ddPCR, droplet digital polymerase chain reaction; 19Del, exon 19 deletion.

\section{Results}

Evaluation of the detection efficiency of ARMS-Plus. Mutant cell lines H1650 (19Del) and H1975 (L858R and T790M) were spiked into EGFR wild-type cell line A549 at a ratio of 1 and $5 \%\left(2 \times 10^{3}\right.$ and $1 \times 10^{4}$ cells of each H1650 and H1975 was spiked into $2 \times 10^{5}$ cells of A549, respectively). QIAamp DNA FFPE Tissue Kit (Qiagen) was used to extract DNA from the cell mixtures. The final allelic frequencies were analyzed by both ARMS-Plus and ddPCR (3 repeats for each cell mixture). Results demonstrated that all mutations were stably detected by ARMS-Plus (Fig. 1). Generally, the mutant abundance detected by ddPCR was higher than that by ARMS-Plus.

Patients' enrollment. FFPE tumor samples from 77 lung adenocarcinoma patients were collected in this study (Table III). Of these patients, 45\% (35/77) were female, median age was 62 years (range from 37-91 years), and 52\% were never-smokers. Nearly half of the patients $(45 \%, 35 / 77)$ were diagnosed with advanced stage disease (stage III-IV). Only 4 patients were treated with EGFR-TKI before sample collection.

Determination of EGFR mutation status. ARMS-Plus was performed to analyze the EGFR mutation status. The results were then re-tested by ddPCR and ARMS-PCR (Table IV).

Among the 77 paired samples evaluated by ddPCR, $35(45.5 \%)$ samples were mutation positive and the other 42 samples were negative. For the ARMS-Plus results, only one sample showed a discordant result compared to that of ddPCR. A case of weak positive $L 858 R$ result with an abundance of $0.5 \%$ detected by ARMS-Plus was recognized as negative by ddPCR (Table V). Together, the overall concordance rate of EGFR mutation testing between ARMS-Plus and ddPCR was $98.7 \%$ (76/77). The results of EGFR mutation status identified by the two methods were highly consistent (Kappa $=0.9739$, 95\% CI: 0.923-1).

To evaluate the utility of ARMS-Plus for identifying $E G F R$ mutations, we assessed the sensitivity and specificity of the assay compared to ddPCR (Table VI). For 19Del and
T790M mutations, ARMS-Plus results were $100 \%$ matched with that of ddPCR. Only one case of $L 858 R$ mutation was detected in ARMS-Plus but not ddPCR. The overall sensitivity and specificity for detecting EGFR mutations were 100 and 98\% respectively, demonstrating the promise of ARMS-Plus in identifying EGFR mutations.

The ARMS-PCR results were also similar to that of ARMS-Plus and ddPCR. Only one case of positive 19Del result confirmed by both ARMS-Plus and ddPCR was classified as negative by ARMS-PCR and one case of negative 19Del result detected by both ARMS-Plus and ddPCR was shown positive for ARMS-PCR. Collectively, these data indicate that both ARMS-PCR, ddPCR and ARMS-Plus results were consistent in identifying EGFR mutations in FFPE-derived DNA samples.

Quantitative analysis of EGFR mutations. 23 out of 77 samples were identified to be 19Del mutant by both ARMS-Plus and ddPCR. According to the results, significant difference was observed (Wilcoxon rank sum test, $\mathrm{P}=0.0002$, Fig. $2 \mathrm{~A}$ ) between the abundance of 19Del mutation detected by ARMS-Plus $($ median $=25.26 \%)$ and ddPCR (median $=43.76 \%)$. The observed 19Del mutant abundance detected by ARMS-Plus was not consistent with the expected abundance based on ddPCR (Linear regression analysis, $R^{2}=0.1012$, Fig. $2 \mathrm{~B}$ ).

For $L 858 R$ mutation, 12 out of 77 samples were identified by both ARMS-Plus and ddPCR. No significant difference was observed (Wilcoxon rank sum test, $\mathrm{P}=0.7334$, Fig. 3A) between the abundance of $L 858 R$ mutation detected by ARMS-Plus (median $=31.18 \%$ ) and ddPCR (median $=26.27 \%$ ). The observed $L 858 R$ mutant abundance detected by ARMS-Plus was highly consistent with the expected abundance based on ddPCR (Linear regression analysis, $R^{2}=0.8081$, Fig. 3B).

The data of $T 790 M$ mutant abundance were not analyzed as only 2 samples were identified with $T 790 M$ mutation (both patients were EGFR-TKI treatment-naïve). However, the observed mutant abundance detected by ARMS-Plus is generally consistent with that of ddPCR (Case 1: 29.21\% and $43.17 \%$; Case 2: 11.18 and $16.42 \%$, for ARMS-Plus and ddPCR, respectively). 
Table III. Patient characteristics.

EGFR mutation status detected by ddPCR

\begin{tabular}{|c|c|c|c|}
\hline \multirow{2}{*}{ Variable } & \multirow[b]{2}{*}{$\mathrm{n}(\%)$} & \\
\hline & & Mutant & Wild-type \\
\hline \multicolumn{4}{|l|}{ Sex } \\
\hline Female & $35(45)$ & 24 & 11 \\
\hline Male & $42(55)$ & 11 & 31 \\
\hline \multicolumn{4}{|l|}{ Age } \\
\hline$\geq 60$ years & $46(60)$ & 15 & 31 \\
\hline$<60$ years & $31(40)$ & 20 & 11 \\
\hline \multicolumn{4}{|l|}{ Smoking history } \\
\hline Ever-smoker & $33(43)$ & 9 & 24 \\
\hline Never-smoker & $40(52)$ & 26 & 14 \\
\hline Uncertain & $4(5)$ & 0 & 4 \\
\hline \multicolumn{4}{|l|}{ Disease stage } \\
\hline I & $7(9)$ & 5 & 2 \\
\hline II & $26(34)$ & 12 & 14 \\
\hline III & $10(13)$ & 5 & 5 \\
\hline IV & $25(32)$ & 12 & 13 \\
\hline Uncertain & $9(12)$ & 1 & 8 \\
\hline \multicolumn{4}{|l|}{ EGFR mutation type } \\
\hline 19Del & $21(27)$ & 21 & 0 \\
\hline$L 858 R$ & $12(16)$ & 12 & 0 \\
\hline 19Del+T790M & $2(3)$ & 2 & 0 \\
\hline Wild-type & $42(54)$ & 0 & 42 \\
\hline \multicolumn{4}{|l|}{ Histopathology type } \\
\hline Adenocarcinoma & $77(100)$ & 35 & 42 \\
\hline Total n & 77 & 35 & 42 \\
\hline
\end{tabular}

$E G F R$, epidermal growth factor receptor; ddPCR, droplet digital polymerase chain reaction; 19Del, exon 19 deletion.

Table IV. EGFR mutation status detected by ARMS-PCR, ddPCR and ARMS-Plus.

\begin{tabular}{lccc}
\hline Mutation & ARMS-PCR & ddPCR & ARMS-Plus \\
\hline Wild type & 43 & 42 & 41 \\
19Del & 20 & 21 & 21 \\
L858R & 11 & 12 & 13 \\
19Del+L858R & 1 & 0 & 0 \\
19Del+T790M & 2 & 2 & 2 \\
Total n & 77 & 77 & 77 \\
\hline
\end{tabular}

$E G F R$, epidermal growth factor receptor; ARMS, amplification refractory mutation system; ddPCR, droplet digital polymerase chain reaction; 19Del, exon 19 deletion.

\section{Discussion}

Since the approval of EGFR-TKIs in the last decade, a massive amount of researches have been investigating the clinical significance of EGFR mutations in predicting the sensitivity
Table V. Comparison of EGFR mutation status detected by ARMS-Plus and ddPCR.

\begin{tabular}{lccc}
\hline & \multicolumn{2}{c}{ ARMS-Plus results } & \\
\cline { 2 - 3 } Variable & EGFR Mutant (n) & Wild-type (n) & Total \\
\hline ddPCR results & & & \\
EGFR Mutant (n) & 35 & 0 & 35 \\
Wild-type (n) & 1 & 41 & 42 \\
Total & 36 & 41 & 77 \\
\hline
\end{tabular}

$E G F R$, epidermal growth factor receptor; ARMS, amplification refractory mutation system; ddPCR, droplet digital polymerase chain reaction.

of TKIs treatment $(22,23)$. It is now apparent that $E G F R$ mutations are associated with the efficacy of TKIs therapy. All NSCLC patients are suggested to perform EGFR mutation test after diagnosis to save cost and avoid unnecessary adverse effects due to wrong regimen decision (24). Recently, clinical 
Table VI. Performance of ARMS-Plus compared with ddPCR.

\begin{tabular}{lccccc}
\hline Mutation & Sensitivity $(\%)$ & Specificity $(\%)$ & PPV $(\%)$ & NPV $(\%)$ & Total n number \\
\hline 19Del & 100 & 100 & 100 & 100 & 23 \\
L858R & 100 & 98 & 92 & 100 & 13 \\
T790M & 100 & 100 & 100 & 100 & 2 \\
Total & 100 & 98 & 97 & 100 & 36 \\
\hline
\end{tabular}

19Del, exon 19 deletion; PPV, positive predictive value; NPV, negative predictive value; ARMS, amplification refractory mutation system; ddPCR, droplet digital polymerase chain reaction.

A

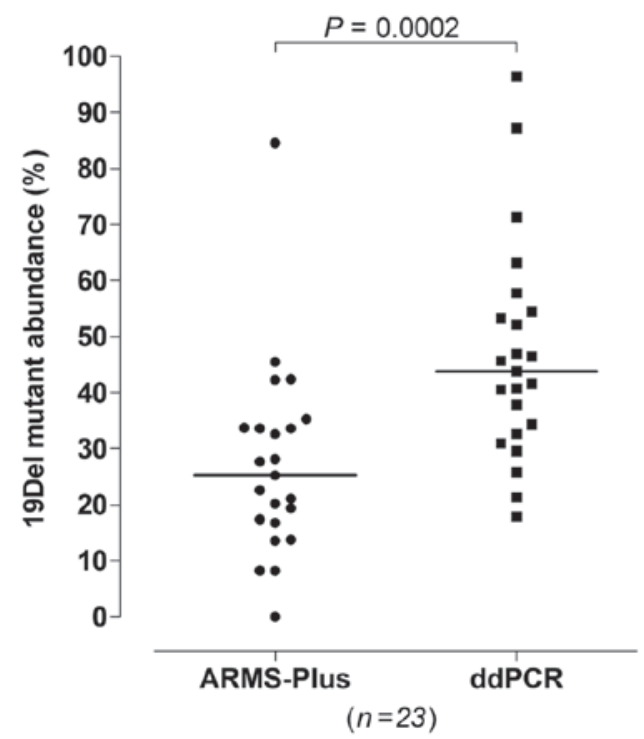

B

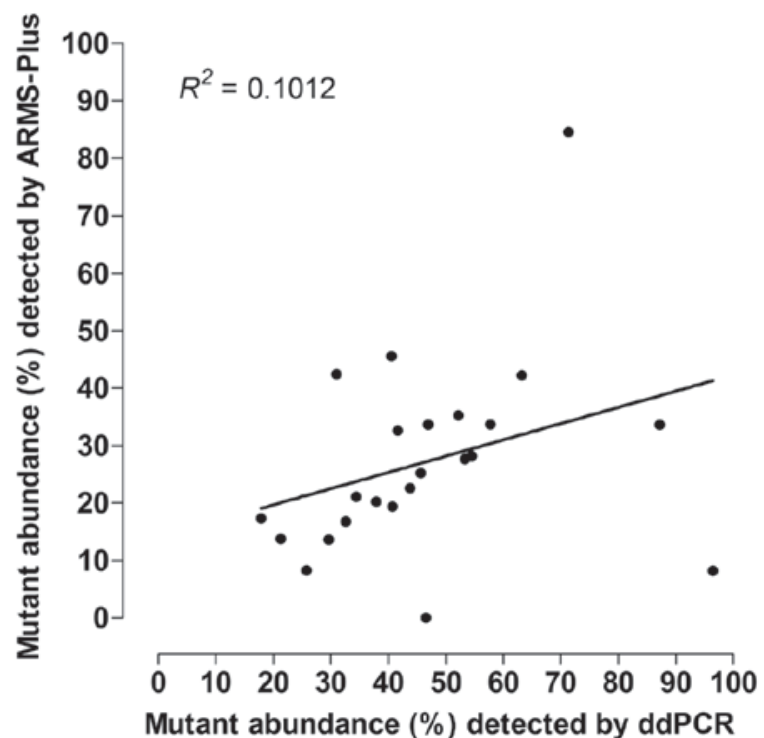

Figure 2. Quantitative analysis of 19Del mutation in paired samples. Comparison of AMRS-Plus and ddPCR results, evaluating 19Del mutant abundance by (A) Wilcoxon rank sum test and (B) linear regression analysis. 19Del, exon 19 deletion; ARMS, amplification refractory mutation system; ddPCR, droplet digital polymerase chain reaction.

A

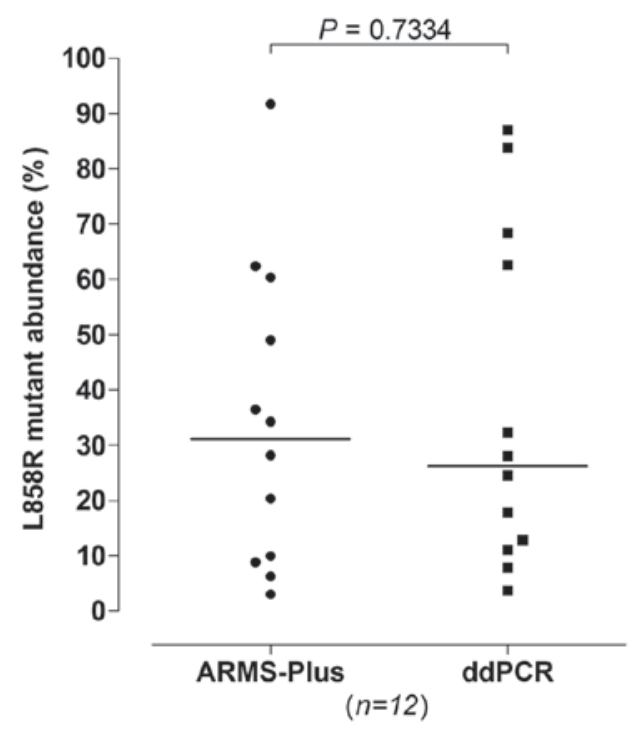

B

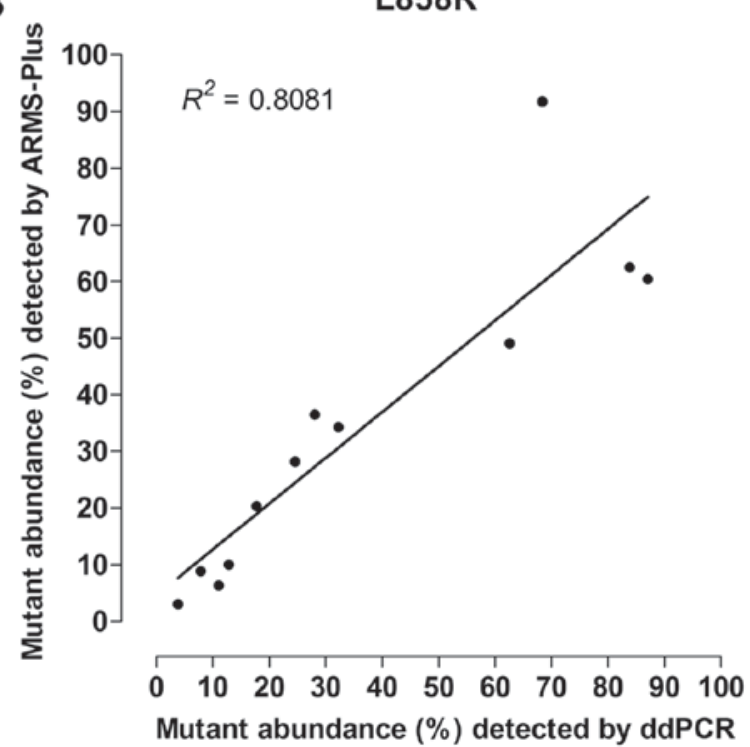

Figure 3. Quantitative analysis of $L 858 R$ mutation in paired samples. Comparison of AMRS-Plus and ddPCR results, evaluating $L 858 R$ mutant abundance by (A) Wilcoxon rank sum test and (B) linear regression analysis. ARMS, amplification refractory mutation system; ddPCR, droplet digital polymerase chain reaction. 
study revealed that Osimertinib, a third-generation EGFR-TKI that can overcome T790M-resistance, showed a superior efficacy as first-line treatment to EGFR-mutated advanced NSCLC compared to first-generation EGFR-TKIs (25). Hence, a sensitive assay that can identify patients with 'de novo' T790M mutation could be valuable for selecting patients who are favorable to first-line Osimertinib treatment.

As NSCLC are often detected at advanced stages, small diagnostic biopsy specimens are the primary source for mutation detection. The DNA amount in these specimens is usually limited due to prior morphologic and immunophenotypic analysis. Thus, a highly sensitive assay is required for the detection of EGFR mutations. ddPCR is now generally accepted as the most sensitive method for evaluating mutations with low mutation rate. It utilizes a water-oil emulsion droplet system to separate DNA samples into thousands of nanoliter-sized droplets. The partitioning enables the measurement of thousands of independent amplification events within a single sample and extensively raises the sensitivity of the assay. In 2014, Zhang et al reported the superiority of ddPCR in detecting EGFR mutations. Results illustrated that ddPCR was able to detect and quantify EGFR mutations for as low as $0.12-2.73 \%$ (26). Despite the high sensitivity of ddPCR, the high cost and the requirement of unique equipment have greatly hampered its clinical application.

In the present study, we interpreted the ability of ARMS-Plus in identifying and quantifying EGFR mutations, with ddPCR as a standard. ARMS-Plus is an AMRS-based assay optimized for the quantification of 4 different EGFR mutations, including $19 \mathrm{Del}$ at two different sites, $L 858 R$, and T790M mutations. Although only limited mutation sites are analyzed by ARMS-Plus, they represent the majority of mutations which have a well-established clinical significance in guiding EGFR-TKIs treatment selection. Moreover, the primers and probes of ARMS-Plus are optimized for EGFR mutation detection and the amplicon of each quantitative PCR is shortened to $50-80 \mathrm{bp}$. These features enable the precise analysis of highly fragmented DNA molecules extracted from FFPE tumor samples. To specifically quantify $E G F R$ mutations, a kind of 3'end-phosphorylated blocking primer was employed into the ARMS-Plus PCR reaction pools. By complementing the wild type DNA at the mutated sites, these primers block the non-specific elongations and raise the accuracy in quantification. In addition, ARMS-Plus can be conveniently performed with a real-time PCR system. These features of ARMS-Plus allow a rapid, reliable and cost-effective molecular analysis in clinical practice.

Cell experiment was performed to evaluate the detection efficiency of ARMS-Plus. EGFR mutations from well-studied cell lines (H1650 and H1975) were stably detected by both ARMS-Plus and ddPCR assays. Generally, the mutant abundance detected by ddPCR were higher than that by ARMS-Plus. This could be due to the possible non-specific amplification of wild-type template in ddPCR.

To explore the sensitivity of ARMS-Plus, a total of 77 FFPE tumor samples were analyzed for EGFR mutation statuses by both ARMS-PCR, ddPCR and ARMS-Plus. Micro-dissection was performed by a pathologist before the tests to remove normal tissues presented in the FFPE samples which could affect the quantification of mutations. All mutations found by ddPCR (35/77) were consistent with the results of ARMS-Plus. Only one case of weak positive L858R mutation with an abundance of $0.5 \%$ was identified by ARMS-Plus, but not by ddPCR and ARMS-PCR. The overall concordance rate of EGFR mutation testing was 98.7\% (Kappa=0.9739). These results demonstrated that ARMS-Plus is a promising assay with high sensitivity in detecting EGFR mutations.

In the quantification of $E G F R$ mutations, the results varied with different types of mutation. For L858R mutation, there was no significant difference $(\mathrm{P}=0.7334)$ between the abundance detected by ARMS-Plus and ddPCR and a clear positive correlation was observed $\left(R^{2}=0.8081\right)$. However, a significant difference was found $(\mathrm{P}=0.0002)$ between the abundance of $19 \mathrm{Del}$ mutation detected by ARMS-Plus and ddPCR. The $19 \mathrm{Del}$ mutant abundance detected by ARMS-Plus was not consistent with the expected abundance based on ddPCR $\left(R^{2}=0.1012\right)$. The duplex probes used in ddPCR for detecting 19Del mutation cover a larger region than that in ARMS-Plus (ddPCR: K749-S752 and A755-D761 region, ARMS-Plus: E746_A750 region) (21). This could probably explain the difference in $19 \mathrm{Del}$ mutant abundance detected by the two methods, where the results of ddPCR were significantly higher than that of ARMS-Plus (median=43.76\% vs. $25.26 \%$ ). Further research with a larger sample size is required to clarify the ability of ARMS-Plus in evaluating 19Del mutant abundance. For $T 790 M$ mutation, because only 2 samples were identified, no analysis is performed to evaluate its correlation between ARMS-Plus and ddPCR.

There were a few limitations in this work. First, FFPE samples can only represents a unique tumor cell clones and cannot reflect the genetic heterogeneity of the whole tumor. The high sensitivity molecular screening for detection of EGFR mutations is at risk of over-qualification for EGFR-TKIs therapy. Therefore, combining liquid biopsy is a better option for decision making. Indeed, ddPCR has been proved to be sensitive enough to detect EGFR mutations in plasma samples (27). Further study to investigate the performance of ARMS-Plus in liquid biopsy is required. Second, due to the small sample size of this study, the performance of ARMS-Plus in quantifying T790M mutation cannot be assessed. Third, although micro-dissection was performed to ensure a $>80 \%$ tumor content in each sample, the variation in tumor content could cause inaccurate quantification.

In conclusion, ARMS-Plus provides a reliable and cost-effective quantitative measuring tool with a similar sensitivity and specificity to ddPCR and could be applicable for evaluating EGFR mutations and guiding regimen selection in lung adenocarcinoma patients.

\section{Acknowledgements}

The authors Dr ZW Guo and Dr GH Yang are employees of GenoSaber Biotech Co., Ltd. (Shanghai, China). This company provided the Human EGFR Gene Mutation Quantitative Detection kit (Fluorescence qPCR) used in the present study and also provided technical support.

\section{References}

1. National Comprehensive Cancer Network (NCCN): Non-small cell lung cancer, Version 8.2017. NCCN Clinical Practice Guidelines in Oncology. NCCN, Fort Washington, PA, 2017. 
2. Boch C, Kollmeier J, Roth A, Stephan-Falkenau S, Misch D, Grüning W, Bauer TT and Mairinger T: The frequency of EGFR and KRAS mutations in non-small cell lung cancer (NSCLC): Routine screening data for central Europe from a cohort study. BMJ Open 3: pii: e002560, 2013.

3. Paez JG, Jänne PA, Lee JC, Tracy S, Greulich H, Gabriel S, Herman P, Kayye FJ, Lindeman N, Boggon TJ, et al: EGFR mutations in lung cancer: Correlation with clinical response to gefitinib therapy. Science 304: 1497-1500, 2004.

4. Rosell R, Carcereny E, Gervais R, Vergnenegre A, Massuti B, Felip E, Palmero R, Garcia-Gomez R, Pallares C, Sanchez JM, et al: Erlotinib versus standard chemotherapy as first-line treatment for European patients with advanced EGFR mutation-positive non-small-cell lung cancer (EURTAC) A multicentre, open-label, randomised phase 3 trial. Lancet Oncol 13: 239-246, 2012.

5. Sequist LV, Yang JC, Yamamoto N, O'Byrne K, Hirsh V, Mok T, Geater SL, Orlov S, Tsai CM, Boyer M, et al: Phase III study of afatinib or cisplatin plus pemetrexed in patients with metastastic lung adenocarcinoma with EGFR mutation. J Clin Oncol 31 $3327-3334,2013$.

6. Mitsudomi T and Yatabe Y: Epidermal growth factor receptor in relation to tumor development: EGFR gene and cancer. FEBS J 277: 301-308, 2010.

7. Fukuoka M, Yano S, Giaccone G, Tamura T, Nakagawa K, Douillard JY, Nishiwaki Y, Vansteenkiste J, Kudoh S Rischin D, et al: Multi-institutional randomized phase II trial of gefitinib for previously treated patients with advanced non-small-cell lung cancer (The IDEAL 1 Trial) [corrected]. J Clin Oncol 21: 2237-2246, 2003.

8. Kris MG, Natale RB, Herbst RS, Lynch TJ Jr, Prager D, Belani CP, Schiller JH, Kelly K, Spiridonidis H, Sandler A, et al: Efficacy of gefitinib, an inhibitor of the epidermal growth factor receptor tyrosine kinase, in symptomatic patients with non-small cell lung cancer: A randomized trial. JAMA 290: 2149-2158, 2003.

9. Usuda K, Sagawa M, Motono N, Ueno M, Tanaka M, Machida Y, Matoba M, Taniguchi M, Tonami H, Ueda Y and Sakuma T: Relationships between EGFR mutation status of lung cancer and preoperative factors-are they predictive? Asian Pac J Cancer Prev 15: 657-662, 2014.

10. Kosaka T, Yatabe Y, Endoh H, Kuwano H, Takahashi T and Mitsudomi T: Mutations of the epidermal growth factor receptor gene in lung cancer: Biological and clinical implications. Cancer Res 64: 8919-8923, 2004.

11. Sutani A,Nagai Y,UdagawaK,Uchida Y,KoyamaN,Murayama Y, Tanaka T, Miyazawa H, Nagata M, Kanazawa M, et al: Gefitinib for non-small-cell lung cancer patients with epidermal growth factor receptor gene mutations screened by peptide nucleic acid-locked nucleic acid PCR clamp. Br J Cancer 95: 1483-1489, 2006.

12. Inoue A, Suzuki T, Fukuhara T, Maemondo M, Kimura Y, Morikawa N, Watanabe H, Saijo Y and Nukiwa T: Prospective phase II study of gefitinib for chemotherapy-naive patients with advanced non-small-cell lung cancer with epidermal growth factor receptor gene mutations. J Clin Oncol 24: 3340-3346, 2006.

13. Shih JY, Gow CH and Yang PC: EGFR mutation conferring primary resistance to gefitinib in non-small-cell lung cancer. N Engl J Med 353: 207-208, 2005.

14. Su KY, Chen HY, Li KC, Kuo ML, Yang JC, Chan WK, Ho BC Chang GC, Shih JY, Yu SL and Yang PC: Pretreatment epidermal growth factor receptor (EGFR) T790M mutation predicts shorter EGFR tyrosine kinase inhibitor response duration in patients with non-small-cell lung cancer. J Clin Oncol 30: 433-440, 2012.
15. Zhou Q, Zhang XC, Chen ZH, Yin XL, Yang JJ, Xu CR, Yan HH, Chen HJ, Su J, Zhong WZ, et al: Relative abundance of EGFR mutations predicts benefit from gefitinib treatment for advanced non-small-cell lung cancer. J Clin Oncol 29: 3316-3321, 2011

16. Zhao ZR, Wang JF, Lin YB, Wang F, Fu S, Zhang SL, Su XD, Jiang L, Zhang YG, Shao JY and Long H: Mutation abundance affects the efficacy of EGFR tyrosine kinase inhibitor readministration in non-small-cell lung cancer with acquired resistance. Med Oncol 31: 810, 2014

17. Ogino S, Kawasaki T, Brahmandam M, Yan L, Cantor M, Namgyal C, Mino-Kenudson M, Lauwers GY, Loda M and Fuchs CS: Sensitive sequencing method for KRAS mutation detection by Pyrosequencing. J Mol Diagn 7: 413-421, 2005.

18. Cushman-Vokoun AM, Crowley AM, Rapp SA and Greiner TC: Comparison study of the performance of the QIAGEN EGFR RGQ and EGFR pyro assays for mutation analysis in non-small cell lung cancer. Am J Clin Pathol 140: 7-19, 2013

19. Shaozhang Z, Ming Z, Haiyan P, Aiping Z, Qitao Y and Xiangqun S: Comparison of ARMS and direct sequencing for detection of EGFR mutation and prediction of EGFR-TKI efficacy between surgery and biopsy tumor tissues in NSCLC patients. Med Oncol 31: 926, 2014.

20. Ellison G, Donald E, McWalter G, Knight L, Fletcher L, Sherwood J, Cantarini M, Orr M and Speake G: A comparison of ARMS and DNA sequencing for mutation analysis in clinical biopsy samples. J Exp Clin Cancer Res 29: 132, 2010.

21. Xu Q, Zhu Y, Bai Y, Wei X, Zheng X, Mao M and Zheng G: Detection of epidermal growth factor receptor mutation in lung cancer by droplet digital polymerase chain reaction. Onco Targets Ther 8: 1533-1541, 2015.

22. Mok T, Wu YL, Lee JS, Yu CJ, Sriuranpong V, Sandoval-Tan J, Ladrera G, Thongprasert S, Srimuninnimit V, Liao M, et al: Detection and dynamic changes of EGFR mutations from circulating tumor DNA as a predictor of survival outcomes in NSCLC patients treated with first-line intercalated Erlotinib and chemotherapy. Clin Cancer Res 21: 3196-3203, 2015.

23. Tseng JS, Yang TY, Tsai CR, Chen KC, Hsu KH, Tsai MH, Yu SL, Su KY, Chen JJ and Chang GC: Dynamic plasma EGFR mutation status as a predictor of EGFR-TKI efficacy in patients with EGFR-mutant lung adenocarcinoma. J Thorac Oncol 10: 603-610, 2015.

24. Lilenbaum RA and Horn LA: Management of EGFR mutation-positive non-small cell lung cancer. J Natl Compr Canc Netw 14 (5 Suppl): S672-S674, 2016.

25. Ramalingam SS, Yang JC, Lee CK, Kurata T, Kim DW, John T, Nogami N, Ohe Y, Mann H, Rukazenkov Y, et al: Osimertinib as first-line tratment of EGFR mutation-positive advanced non-small-cell lung cancer. J Clin Oncol: Aug 25, 2017 (Epub ahead of print)

26. Zhang BO, Xu CW, Shao Y, Wang HT, Wu YF, Song YY, Li XB Zhang Z, Wang WJ, Li LQ and Cai CL: Comparison of droplet digital PCR and conventional quantitative PCR for measuring EGFR gene mutation. Exp Ther Med 9: 1383-1388, 2015.

27. Zhu G, Ye X, Dong Z, Lu YC, Sun Y, Liu Y, McCormack R, Gu Y and Liu X: Highly sensitive droplet digital PCR method for detection of EGFR-activating mutations in plasma cell-free DNA from patients with advanced non-small cell lung cancer. J Mol Diagn 17: 265-272, 2015.

c) (7) $\odot$ This work is licensed under a Creative Commons Attribution-NonCommercial-NoDerivatives 4.0 International (CC BY-NC-ND 4.0) License. 